\title{
Modelos de financiación de la educación superior estatal: El caso de la Universidad de Costa Rica
}

Financing models of the higher public education: the case of the University of Costa Rica

\section{Volumen 18, Número 1 \\ Enero-Abril}

pp. 1-23

\section{Este número se publica el $1^{\circ}$ de enero de 2018}

DOI: https://doi.org/10.15517/aie.v18i1.31852

Carlos Araya Leandro

Revista indizada en REDALYC, $\underline{\text { SCIELO }}$

Revista distribuida en las bases de datos:

LATINDEX, DOAJ, REDIB, IRESIE, CLASE, DIALNET, SHERPA/ROMEO, QUALIS-CAPES, MIAR

Revista registrada en los directorios:

ULRICH'S, REDIE, RINACE, OEI, MAESTROTECA, PREAL, $\underline{\text { CLACSO }}$ 


\title{
Modelos de financiación de la educación superior estatal: El caso de la Universidad de Costa Rica \\ Financing models of the higher public education: the case of the University of Costa Rica
}

\section{Carlos Araya Leandro ${ }^{1}$}

\begin{abstract}
Resumen: En el presente artículo de revisión bibliográfica, se analiza el modelo de financiación de la educación superior estatal en Costa Rica. Como resultado, de la difícil situación económica por la que atraviesan la mayoría de países del mundo y de una mayor demanda por educación superior, en los últimos años se han desarrollado diversos modelos para el financiamiento de la educación superior pública estatal. En el caso particular de Costa Rica, en años recientes, el Estado ha apostado por una mayor participación en el financiamiento de la educación en todos sus niveles. Bajo este contexto, el presente artículo pretende analizar el modelo de financiación de la educación superior pública estatal vigente en Costa Rica, en general, y en la Universidad de Costa Rica, en particular. El estudio desarrollado es de carácter descriptivo; para tal efecto, se ha procedido a realizar una búsqueda de datos cuantitativos, cualitativos y normativos que permitan hacer un recuento del desarrollo del modelo de financiamiento vigente y de sus principales características. Con respecto a esta materia, es poco lo que se ha publicado en el país, de manera que el estudio llena un vacío al sistematizar información dispersa para el análisis de un tema de alta relevancia en las políticas públicas costarricenses. En este sentido, la doble reforma del artículo 78 de la Constitución Política en 1997 y 2011, que obliga al Estado a invertir en educación, en primera instancia un $6 \%$ del Producto Interno Bruto (PIB) y posteriormente el $8 \%$ de ese indicador, ha permitido que el presupuesto de las universidades estatales costarricenses se haya incrementado de manera considerable y sostenida, pues pasa de representar el 0,80\% del PIB en 1999 a un 1,42\% en 2017.
\end{abstract}

Palabras clave: educación superior, financiamiento de la educación, Universidad de Costa Rica

\begin{abstract}
In the bibliographic article, we analyze the financing model of state higher education in Costa Rica. As a result, not only from the difficult economical situation, in wich most of the world countries have been going through but also a higher demand for a higher education in the last years, there have been developed several models for giving economical support to the higher public state education. In the particular case of Costa Rica, some years ago, the state has bet for a higher participation in the economical support to the education in all its levels. In this context, this article pretends to analyze the model for an economical support to the current higher public state education in Costa Rica in general and particularly at the University of Costa Rica. The developed study has a descriptive character; for this purpose it has been done a research in terms of quantitative, qualitative and normative data, which allows to make a recount of the development of the current economical support model and the main characteristics of it. On this matter, there are not enough publications about it, so this study fills the gap by systematizing the dispersed information for the analysis of a high importance subject in terms of the Costa Rican public policies. In this way, the double reform of the article 78 of the Political Constitution in 1997 and 2011, which forces the state to take action in the education, beginning with a $6 \%$ of the Gross Domestic Product (GDP) and then with an $8 \%$ of that indicator, they have allowed the budget of the Costa Rican state universities to increase considerably and continuously from a 0,80\% of the GDP in 1999 to a 1,42\% in 2017.
\end{abstract}

Key words: higher education, educational finance, University of Costa Rica

\footnotetext{
${ }_{1}^{1}$ Docente e investigador de la Universidad de Costa Rica, actualmente se desempeña en el puesto de Vicerrector de Administración de esta Universidad. Doctor en Economía, Gestión y Control de entidades y políticas públicas por la Universidad de Granada, España.
}

Dirección electrónica carlos.arayaleandro@ucr.ac.cr

Artículo recibido: 5 de mayo, 2017

Enviado a corrección: 2 de noviembre, 2017

Aprobado: 18 de diciembre, 2017 


\section{Introducción}

Uno de los principales vehículos para la movilidad social y para el desarrollo de los países es la educación. En este sentido, el sistema educativo ha ocupado un papel preponderante en las políticas públicas del Estado costarricense, sobre todo, a partir de la década de los años cuarenta del siglo XX. La educación superior no ha sido la excepción. En primer término, con la fundación de la Universidad de Costa Rica en el año 1940; posteriormente, con la creación de tres universidades en la década de los años setenta y por último, con la consolidación del sistema de financiamiento. A finales de la década de los años ochenta, se implementa la utilización de convenios de financiamiento como instrumento para acordar y estimar los recursos asignados a las universidades estatales. Si bien, en una primera etapa, este mecanismo de financiamiento generó una caída en la inversión en educación superior respecto al Producto Interno Bruto (PIB), lo cierto del caso es que, en los últimos 15 años, el incremento en el financiamiento es verdaderamente relevante, se pasa de un $0,8 \%$ en 1999 a un $1,42 \%$ del PIB en 2016.

A la fecha de elaboración del presente trabajo se han firmado cinco convenios de financiamiento que, sobre todo en los últimos años, han permitido mejorar de manera sustancial las actividades de docencia, investigación y extensión en las universidades estatales costarricenses. De igual forma, han generado incrementos importantes en la inversión en infraestructura, equipamiento y en el sistema de becas estudiantiles.

A pesar de lo anterior, los recursos no son suficientes para el desarrollo de la actividad sustantiva de estas instituciones, de ahí que estas realicen esfuerzos por generar recursos producto de la vinculación remunerada con el sector externo. En el caso particular de la Universidad de Costa Rica, su Consejo Universitario emitió una serie de lineamientos para la regulación y promoción de la obtención de recursos por esta vía.

La conceptualización de ese sistema de vinculación remunerada con el sector externo tiene un componente de solidaridad académica importante, por cuanto los proyectos de generación de recursos están en la obligación de contribuir con un 15\% de sus ingresos a un Fondo de Desarrollo Institucional que, entre otros objetivos, persigue el financiamiento de proyectos de investigación y acción social en aquellas áreas en las que, por su naturaleza, la vinculación remunerada con el sector externo no es sencilla.

En este contexto, en el presente documento se pretende analizar el modelo de financiación de la educación superior pública estatal vigente en Costa Rica en general, y en la Universidad de Costa Rica, en particular. Para tal efecto, se describirá el modelo de 
financiación de la educación superior estatal en Costa Rica y su comportamiento durante los últimos años. Asimismo, se realizará una valoración de las fuentes y mecanismos de financiación ordinaria en la Universidad de Costa Rica, y la financiación vía vinculación remunerada con el sector externo como fuente alternativa de recursos en esta Universidad, se les brindará especial atención a los mecanismos operativos desarrollados y a la importancia que esta fuente de recursos económicos ha alcanzado en esta casa de estudios.

Se considera importante evidenciar las acciones desarrolladas en el país, en general, y en la Universidad de Costa Rica en particular. De modo que estas puedan servir como base de análisis y comparación para otros países de la región.

\section{La financiación de la educación superior}

Como resultado de diferentes factores, con el nuevo milenio, han brotado gran cantidad de nuevas ideas sobre la financiación de la educación superior. De un lado, la situación económica de muchos de los países del orbe, sobre todo a partir de la crisis económica desatada en el 2008, ha generado que las posibilidades financieras de los diferentes Estados se hayan visto limitadas. De otro lado, la amplia expansión de la educación superior provoca una drástica reducción del presupuesto educativo en cuanto a inversión por estudiante. De esta manera, en términos generales, la contribución económica del Estado para el financiamiento de la educación superior ha tendido a la disminución (Barquero, Calvo, Ortiz, Valverde y Wachong, 2006, p. 42).

Como señalan Sanyal y Martín, hay signos claros de que la expansión de la educación superior continuará a ritmo acelerado en los países en vías de desarrollo. Dado que la expansión masiva no se puede igualar con la correspondiente expansión de la financiación estatal, en todos los países del mundo, empieza a aparecer la financiación del sector privado aplicada a la educación superior (Sanyal y Martín, 2006, p. 12).

Teniendo en cuenta la inhabilidad de los gobiernos para soportar el peso de los gastos, algunos se han traspasado a los padres y al alumnado con la aparición del fenómeno de la distribución de costos, a través de la introducción y del aumento de tasas de matrícula y la eliminación de las subvenciones y de los sistemas de becas o con la introducción de préstamos a estudiantes (Fernández y Fernández, 2007, p. 9).

No obstante, como lo señalan Barquero et al., es preciso comprender que el papel del Estado en la educación superior pública debe ser primordial, especialmente en el financiamiento de su provisión, esto por cuanto la educación superior permite incrementar las 
posibilidades de obtener ingresos y mejora las oportunidades de empleo a las personas que la reciben. La inversión en educación fomenta la construcción de una sociedad más justa y conformada por ciudadanos con mayores capacidades de alcanzar una mejor calidad de vida, más responsables, críticos y activos en los sistemas sociales y políticos (Barquero et al., 2006, p. 61).

Tampoco debe olvidarse que las universidades están en la necesidad de diversificar sus fuentes de ingresos, por lo que deben incluir alternativas provenientes de la vinculación con el sector productivo. De igual forma, es fundamental que estas instituciones tengan altos niveles de eficiencia en el empleo de los recursos asignados y ejerzan una política de transparencia y rendición de cuentas ante la sociedad.

En el caso costarricense, el desarrollo reciente de la financiación de educación superior ha sido contrario a esa tendencia y, a pesar de la difícil situación de las finanzas públicas por la que atraviesa el Estado, se han realizado importantes esfuerzos por aumentar la participación estatal en el financiamiento de todos los niveles educativos. Ya en el 2007, Fernández y Fernández consideraban el caso costarricense como pionero en los procesos de planificación e implantación de modelos formales de financiación de educación superior en la región latinoamericana (Fernández y Fernández, 2007, p. 4).

Según el V Informe del Estado de la Educación (Estado de la Nación, 2016, p. 183), en Costa Rica, hay 208612 estudiantes inscritos en la educación superior, con lo cual la cobertura de la población en edad de asistir (18 a 24 años) a este nivel educativo alcanza el $34,2 \%$. De esta cantidad de estudiantes, poco menos del $50 \%$ asiste a instituciones de educación superior de carácter estatal, las que han incrementado la cantidad de estudiantes matriculados en un $50,6 \%$ en los últimos 15 años.

\section{El sistema de educación superior público estatal en Costa Rica}

El sistema de educación superior público estatal en Costa Rica está compuesto por cinco universidades, las cuales se agrupan mediante un ente coordinador denominado el Consejo Nacional de Rectores (Conare), creado mediante el "Convenio de Coordinación de la Educación Superior Universitaria Estatal en Costa Rica”, suscrito por estas Instituciones. La Tabla 1 detalla el nombre de cada una de las Universidades estatales de Costa Rica, su fecha de creación y cantidad de estudiantes al año 2015. 
Tabla 1

Costa Rica: Año de fundación y número de estudiantes por universidad estatal en el año 2015

\begin{tabular}{lcc}
\hline Institución & Año de fundación & № de estudiantes \\
\hline Universidad de Costa Rica & 1940 & 40585 \\
Instituto Tecnológico de Costa Rica & 1971 & 11284 \\
Universidad Nacional de Costa Rica & 1973 & 19189 \\
Universidad Estatal a Distancia & 1977 & 20147 \\
Universidad Técnica Nacional & 2008 & 9804 \\
\hline Total de Estudiantes en Educación Superior Estatal & & 101009 \\
\hline
\end{tabular}

Fuente: Elaboración propia a partir de información disponible en los sitio web de la Universidad de Costa Rica (s.f.), del Instituto Tecnológico de Costa Rica (2016), de la Universidad Nacional de Costa Rica (s.f.), de la Universidad Estatal a Distancia (2015) y de la Universidad Técnica Nacional (2015).

El Conare está formado por los Rectores de las Instituciones signatarias del Convenio y tiene, entre otras funciones, las siguientes:

a) Aprobar el Plan Nacional de la Educación Superior (PLANES), según lo establecido en el artículo 85 de la Constitución Política de la República, que señala: "El cuerpo encargado de la coordinación de la Educación Superior Universitaria Estatal preparará un plan nacional para esta educación, tomando en cuenta los lineamientos que establezca el Plan Nacional de Desarrollo vigente" (Costa Rica, s.f., p. 11).

b) Distribuir las rentas globales asignadas a la Educación Superior Universitaria Estatal en forma congruente con los criterios que se señalan en el Capítulo III del Convenio de articulación, para el cumplimiento del Plan Nacional de Educación Superior Universitaria Estatal, sin perjuicio de que cada Institución reciba, separadamente, las rentas que legalmente le correspondan.

c) Impulsar y fortalecer la coordinación entre las oficinas de programación o el equivalente propio de cada Institución signataria.

d) Recomendar la adopción de políticas comunes, en lo académico y en lo administrativo, por parte de las Instituciones signatarias. (Conare, 2013, pp. 73-74)

Según lo señalado en el Plan Nacional de la Educación Superior 2016-2020:

[...] mediante la coordinación del Conare, las cinco universidades estatales procuran articularse como una vía de doble tránsito que les permita establecer múltiples interrelaciones en las áreas de docencia, investigación, extensión y acción social. Este proceso se nutre de la responsabilidad social de las instituciones y permite optimizar la calidad del quehacer universitario mediante un aporte innovador al desarrollo nacional. Lo anterior, con el fin de afirmar sus identidades, potencialidades y fortalezas dentro de 
un espacio de acción conjugada y un uso racional de los recursos asignados [...] (Conare, 2015, p. 39)

\section{Financiamiento de la educación estatal en Costa Rica}

\subsection{Educación pública en general}

Uno de los principales vehículos del desarrollo social en Costa Rica lo ha representado la educación. Según Martínez, la educación en Costa Rica fue el principal instrumento social con el que se buscaba solidificar la identidad nacional y con el que se pretendían afianzar las relaciones entre las clases sociales. La inversión pública en educación tuvo un crecimiento sostenido a partir de la década de los años cuarenta, con la creación de la Universidad de Costa Rica y la promulgación del Código de la Educación en 1944 (Martínez, 2016, p. 27).

Entre los años 1949 y 1980, Costa Rica tiene un crecimiento muy positivo en términos de desarrollo social e institucional, con lo cual la educación juega un papel preponderante. Por ejemplo, los cambios fundamentales en la escolarización primaria completa de la población costarricense se realizaron claramente a partir del nuevo papel del Estado en la sociedad costarricense, que arranca después de la Guerra Civil del año 1948. De manera privilegiada, en la década de los años setenta, y, en general, a lo largo de este período, todos los indicadores educativos vivieron un sustancial progreso.

Así, el presupuesto del Ministerio de Educación Pública alcanzó el 5,73\% del PIB en 1979 (Fernández y Fernández, 2007, p. 6). Esta tendencia se ve paralizada por la crisis de la década de los años ochenta, ya que en este periodo el gasto público en educación sufrió una caída extraordinaria que osciló entre el 4,75\% y el 4,04\% del PIB (cifras que corresponden a los años 1981 y 1989, respectivamente).

No obstante, según señalan Sauma y Trejos la profunda reducción en la participación del Estado en la financiación de la educación durante esos años de crisis económica, empieza a variar en 1997 con la aprobación de una reforma del artículo 78 de la Constitución Política de la República, la cual establece que el Estado invertirá el equivalente al $6 \%$ del PIB en la educación pública (2014, p. 17).

Posteriormente, en el año 2011 se reforma nuevamente el artículo 78 de la Constitución Política. La reforma establece:

En la educación estatal, incluida la superior, el gasto público no será inferior al ocho por ciento (8\%) anual del producto interno bruto, de acuerdo con la ley, sin perjuicio de lo establecido en los artículos 84 y 85 de esta Constitución. (Costa Rica, s.f., p. 10) 
De igual forma, se señala que el Estado incrementará el porcentaje de inversión en educación de forma gradual, consecuentemente, tendría que alcanzar la cifra del 8\% para el año 2014. Sin embargo, debido a la frágil situación fiscal por la que atraviesa el país, al año 2016, no se ha alcanzado ese porcentaje de inversión, tal y como se detalla en la figura 1.

Figura 1

Costa Rica: Porcentaje de la inversión estatal en educación por año.

Periodo 2008-2016

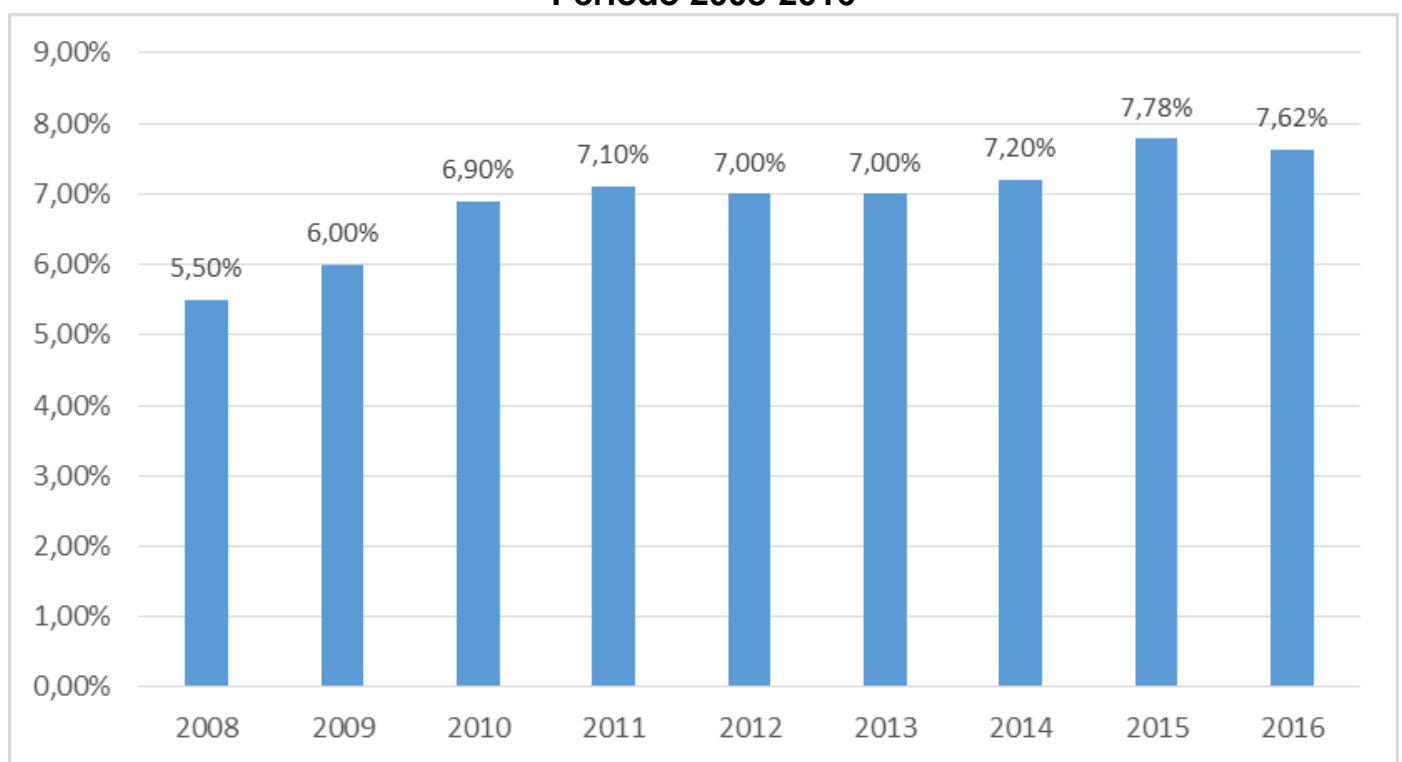

Fuente: Elaboración propia a partir de la información suministrada por la Jefa de la Oficina de Desarrollo Institucional del Conare, Mag. Xinia Morera González (comunicación personal, 21 de febrero, 2017).

A pesar de lo anterior, tal y como se aprecia en la Figura 1, el Estado costarricense ha realizado un esfuerzo relevante en materia de inversión en educación, se pasa del 5,5\% del PIB en 2008 al 7,62\% en 2016. Es importante acotar que la disminución reflejada en la figura entre los años 2015 y 2016 obedece a una nueva base de cálculo del PIB que entró en vigencia el 01 de enero de 2016 y que estableció un monto del PIB mayor al estimado con la fórmula anterior. En términos nominales, la inversión en educación aumentó para 2016 en un $7,32 \%$ respecto al 2015.

En comparación con países de la región latinoamericana, el monto destinado al financiamiento de la educación en Costa Rica se puede considerar alto. La Figura 2 compara los porcentajes de inversión estatal en educación en los países centroamericanos; en los países latinoamericanos, que forman parte de la Organización para Cooperación y el Desarrollo Económico (OCDE); y el promedio de inversión pública en educación en relación 
con el PIB de los países miembros de esta organización. Como puede observarse, el monto destinado a educación en Costa Rica es superior al del resto de países centroamericanos y está aún por encima del promedio de los países que conforman la OCDE, que en su mayoría son considerados como los de niveles de desarrollo más avanzado en el mundo.

Figura 2

Inversión Estatal en Educación como porcentaje del PIB

en los países centroamericanos y algunos de la OCDE.

Cifras en porcentajes respecto al PIB del año 2014

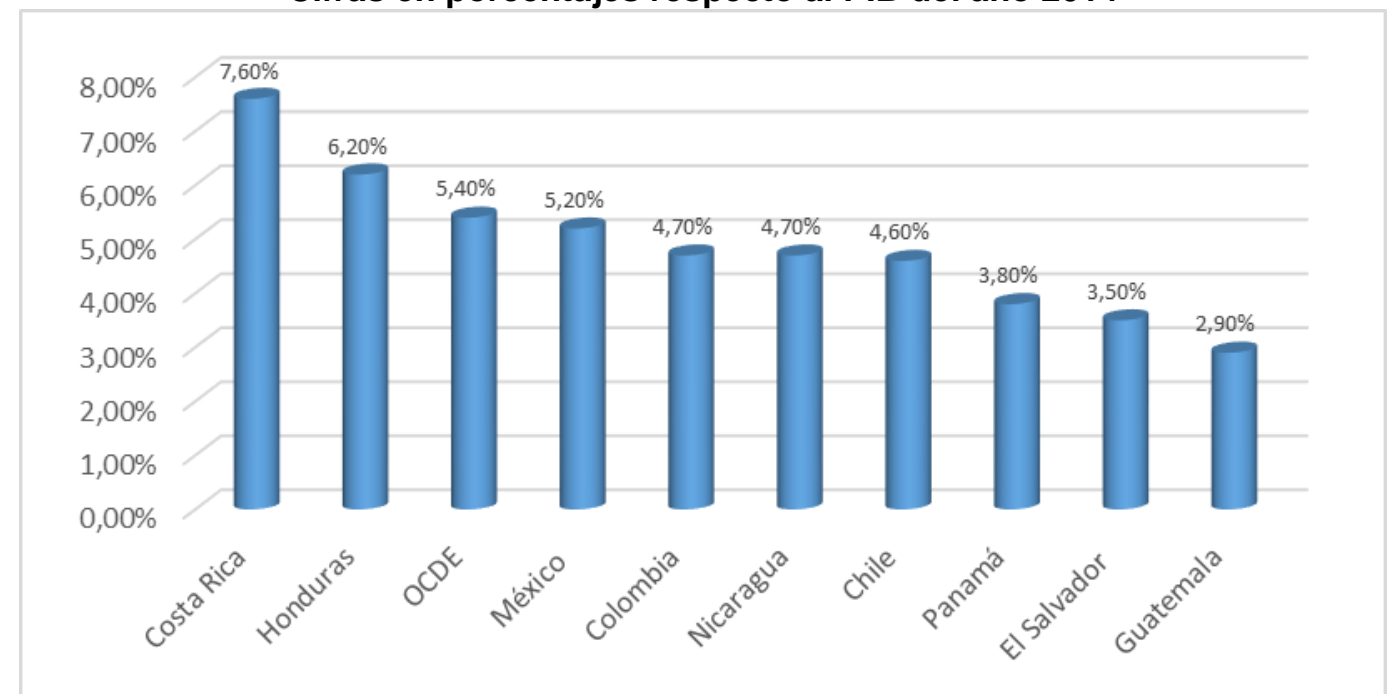

Fuente: Elaboración propia a partir de datos del V Informe del Estado de la Región (2016) y de la OCDE (s.f.).

\subsection{Financiamiento de la Educación Superior}

Dos artículos de la Constitución Política son fundamentales en la definición del modelo de financiación de la educación superior pública en Costa Rica. De un lado, el artículo 78 citado en el punto anterior. De otro lado, el artículo 85 que señala:

[...] El Estado dotará de patrimonio propio a la Universidad de Costa Rica, al Instituto Tecnológico de Costa Rica, a la Universidad Nacional y a la Universidad Estatal a Distancia y les creará rentas propias, independientemente de las originadas en estas instituciones. Además, mantendrá - con las rentas actuales y con otras que sean necesarias - un fondo especial para el financiamiento de la Educación Superior Estatal. El Banco Central de Costa Rica administrará ese fondo y, cada mes, lo pondrá, en dozavos, a la orden de las citadas instituciones, según la distribución que determine el cuerpo encargado de la coordinación de la educación superior universitaria estatal. Las rentas de ese fondo especial no podrán ser abolidas ni disminuidas, si no se crean, simultáneamente, otras mejoras que las sustituyan [...]. (Costa Rica, s.f., p. 11) 
En el momento en que se incluye la redacción de este artículo de la Constitución Política de la República, no se había creado la Universidad Técnica Nacional, razón por la que esta Universidad cuenta con un modelo de financiamiento diferente: no forma parte del Fondo Especial para la Educación Superior (FEES) establecido constitucionalmente.

En cumplimiento de lo señalado en el artículo 85 de la Constitución Política, mediante Ley № 5909 de junio de 1976, se establece el Fondo Especial para el Financiamiento de la Educación Superior. Según dicha Ley, el Banco Central de Costa Rica hará las separaciones correspondientes para la formación del Fondo, el cual será distribuido conforme a las normas y principios establecidos en el Convenio de Coordinación de la Educación Superior en Costa Rica.

EI FEES está constituido por el total de transferencias con destino global que hayan sido acordadas, para la Educación Superior Universitaria Estatal, entre el Gobierno de la República y los Rectores de las universidades estatales.

Con el fin de brindar una base de mayor solidez y definición al aporte estatal para las universidades públicas, a partir del año 1988, se establece un mecanismo diferente para la determinación del FEES. Es así como se crea una Comisión de Enlace, responsable de la negociación del financiamiento a las universidades estatales por periodos quinquenales. La Comisión de Enlace está integrada por los rectores de las instituciones de educación superior pública estatal y los ministros de: Educación Pública, Planificación y Política Económica, Hacienda y Ciencia y Tecnología. Una vez determinado el monto del FEES, este se incluye en el presupuesto del Ministerio de Educación Pública como Transferencias Corrientes al Sector Público. Este mecanismo de financiación se mantiene vigente a la fecha de elaboración de este documento.

En total se han suscrito cinco convenios de financiación, los que se detallan en la Tabla 2.

Tabla 2

Costa Rica: Convenios de financiación de la educación superior aprobados al año 2017

\begin{tabular}{lll}
\hline Convenio & Vigencia & Aprobación \\
\hline Primero & $1989-1993$ & 08 de noviembre 1988 \\
Segundo & $1994-1998$ & 03 de agosto 1993 \\
Tercero & $1999-2004$ & 07 de diciembre 1998 \\
Cuarto & $2005-2009$ & 29 de julio 2004 \\
\hline
\end{tabular}

Fuente: Elaboración propia a partir de la información suministrada por la Jefa de la Oficina de Desarrollo Institucional del Conare, Mag. Xinia Morera González (comunicación personal, 21 de febrero, 2017). 
El primer convenio de financiamiento estableció los procedimientos y una fórmula para el cálculo del FEES; además, señala que los proyectos específicos que requirieran de recursos adicionales deberían ser analizados y aprobados en el seno de la Comisión de Enlace. Básicamente, la fórmula de cálculo establecía como mecanismo de actualización del monto del FEES, el Índice de Precios al Consumidor o tasa de inflación anual. Por las elevadas tasas de inflación de la época y por utilizarse como base de cálculo el monto del FEES del año anterior, la inversión estatal en educación superior como porcentaje del PIB disminuyó en el quinquenio.

El segundo convenio mantuvo la fórmula de cálculo con base en la tasa de inflación, con la diferencia de que, complementariamente, el Gobierno asumió el compromiso de destinar en forma adicional la suma anual del $2 \%$ del FEES del año respectivo para un programa de renovación del equipo científico tecnológico. Además, las Universidades se comprometieron a realizar esfuerzos para generar recursos propios que no dependieran del presupuesto de la República.

El tercer convenio de financiamiento de la educación superior estatal modificó en forma significativa la fórmula de ajuste automático del FEES, agregando además de la tasa de inflación dos variables; a saber: la tasa anual de crecimiento de la población y un factor de participación en el crecimiento económico. Con esta nueva fórmula se mejoran las condiciones de financiamiento respecto a las pactadas en el primer y segundo convenios, incrementándose levemente la inversión en educación superior estatal en relación con el PIB.

Por su parte, el cuarto convenio de financiación modificó radicalmente los procedimientos y la fórmula para el cálculo del FEES en relación con los convenios anteriores. Se deja de utilizar la tasa de inflación como elemento central de los ajustes y el FEES se liga directamente al producto interno bruto nominal. De esta manera, el convenio establece que, en el quinquenio, el porcentaje del PIB invertido en educación superior estatal pasará de 0,90\% para el 2005 al 1,05\% en 2009. Este convenio se amplió por un año más, llegando el monto del FEES a representar, en 2010, el equivalente al 1,19\% del PIB.

Finalmente, el Quinto Convenio FEES se firma en el contexto de la reforma constitucional del artículo 78, que establece la meta de que el Estado invierta el $8 \%$ del PIB en educación. En este marco, se acuerda que en el momento en que se cumpla con esa meta, a la educación superior le corresponderá una inversión equivalente al 1,5\% del PIB. El acuerdo señala lo siguiente: 
[...] En los primeros dos años, el FEES crecerá una tasa anual real garantizada de 7\%, y la tasa garantizada de aumento real para los años subsiguientes será de $4.5 \%$ anual. El financiamiento del FEES aumentará progresivamente del 2013 al 2015 hasta alcanzar en el 2015 el 1,5\% del PIB, en el marco del incremento del 8\% del PIB destinado a la educación y en relación directa con el mejoramiento de la carga tributaria. Para ello el Gobierno de la República establecerá una estrategia de política fiscal que permita una mayor progresividad y justicia tributaria y que la carga tributaria ascienda de su nivel actual de 13,4\% del PIB a 15,9\%, para fortalecer las capacidades de la economía, para crecer en forma equitativa y sostenible y, en particular, para asegurar los recursos necesarios que permitan ir cumpliendo la meta de hacer llegar el FEES al 1,5\% del PIB en el quinquenio [...]. (Conare, 2013, p. 196)

Adicionalmente, este convenio establece que, con el objeto de fortalecer los procesos de inversión en áreas estratégicas de las cuatro instituciones, el Gobierno de la República se compromete a tramitar y financiar, en su totalidad, a favor de las cuatro instituciones que forman en ese momento el Conare y, por ende, reciben financiamiento del FEES, una operación de crédito por 200 millones de dólares, la cual se ejecutará durante el quinquenio.

Es importante resaltar que el alcanzar la meta señalada en el quinto convenio FEES quedaba sujeta a que el Gobierno de la República lograra incrementar la carga tributaria por la vía de creación de nuevos impuestos. No obstante, la reforma tributaria no tuvo éxito y el nivel de carga impositiva respecto al PIB se ha mantenido en rangos que no superan el $13,3 \%^{2}$, lo que ha generado un importante déficit fiscal que no le ha permitido al Gobierno cumplir con lo establecido en el artículo 78 de la Constitución Política.

Siguiendo el esquema de convenios quinquenales, el sexto acuerdo debió regir para los años comprendidos entre 2016 y 2020, sin embargo, producto de la difícil situación fiscal que atraviesa el Estado costarricense, para los años 2016 y 2017 se han suscrito adendas al quinto convenio FEES aprobado. La figura 3 detalla la magnitud del déficit fiscal que arrojan las finanzas públicas en Costa Rica. Como se puede apreciar, durante los últimos siete años se han generado déficits anuales que oscilan entre el $4 \%$ y $5,7 \%$ del PIB, situación que disminuye considerablemente las posibilidades de inversión de parte del Estado costarricense.

2 Datos suministrados por el Ministerio de Hacienda, Costa Rica 
Figura 3

Costa Rica: Déficit fiscal como porcentaje del PIB

para los años comprendidos entre 2010 y 2016

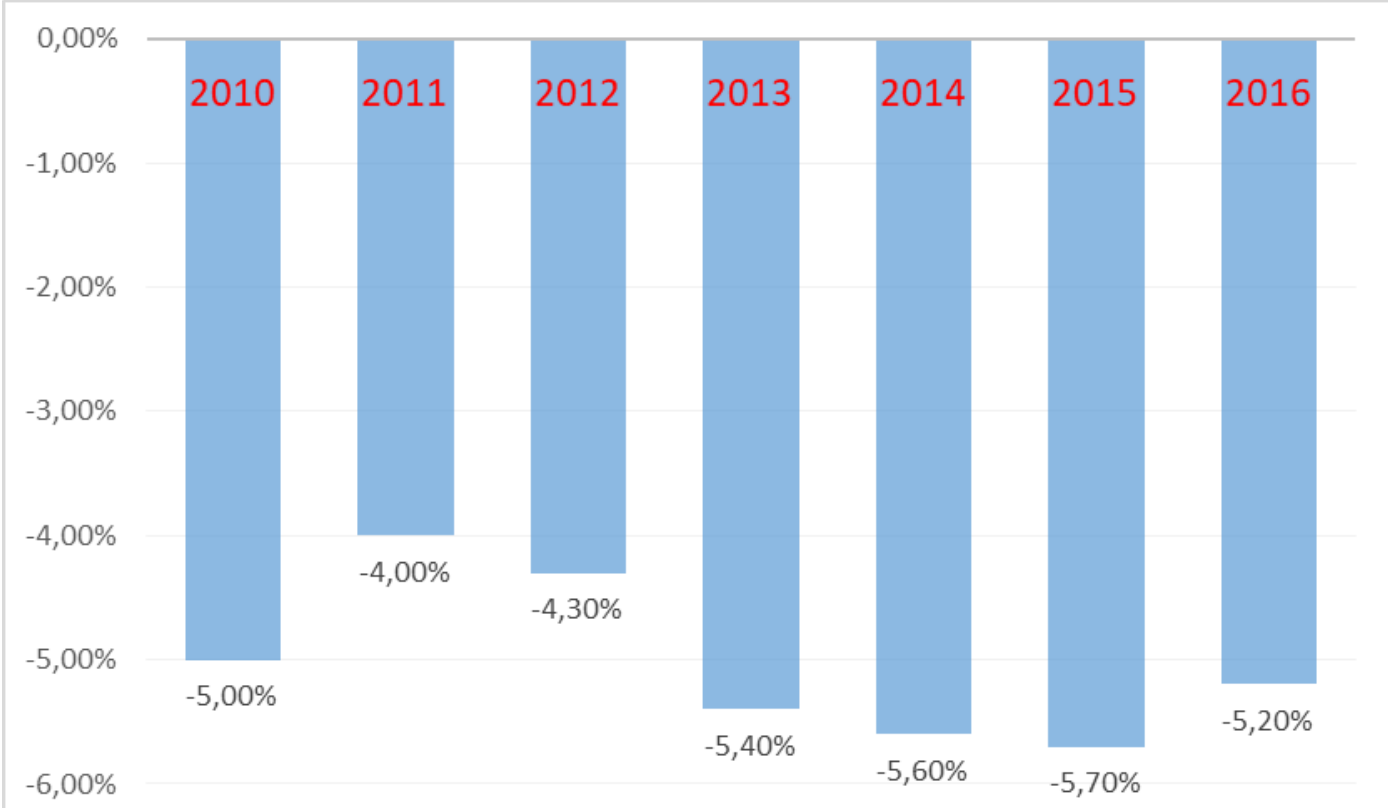

Fuente: Elaboración propia a partir de la información suministrada por el señor Ministro de Hacienda de Costa Rica, M.Sc. Helio Fallas Venegas (comunicación personal, 18 de abril, 2017).

A pesar de la situación deficitaria de las finanzas públicas, lo cierto del caso es que el Gobierno costarricense ha realizado un esfuerzo verdaderamente importante por aumentar la inversión en educación en general y en educación superior estatal en particular. La Figura 4 muestra el comportamiento de la inversión estatal en educación superior pública estatal desde la firma del primer convenio FEES. Como se observa, para el año 2017 la inversión en educación superior alcanza el 1,42\% del PIB. Es importante reiterar que la caída en el porcentaje de inversión, que muestra la gráfica entre los años 2015 y 2016, obedece a una variación en la base de cálculo del PIB. Pese a ello, en dicho período, la inversión en educación superior se incrementó en términos nominales en un 7,32\%. 
Figura 4

Costa Rica: Financiación de la Educación Superior Pública como porcentaje del PIB para los años comprendidos entre 1989 y 2017

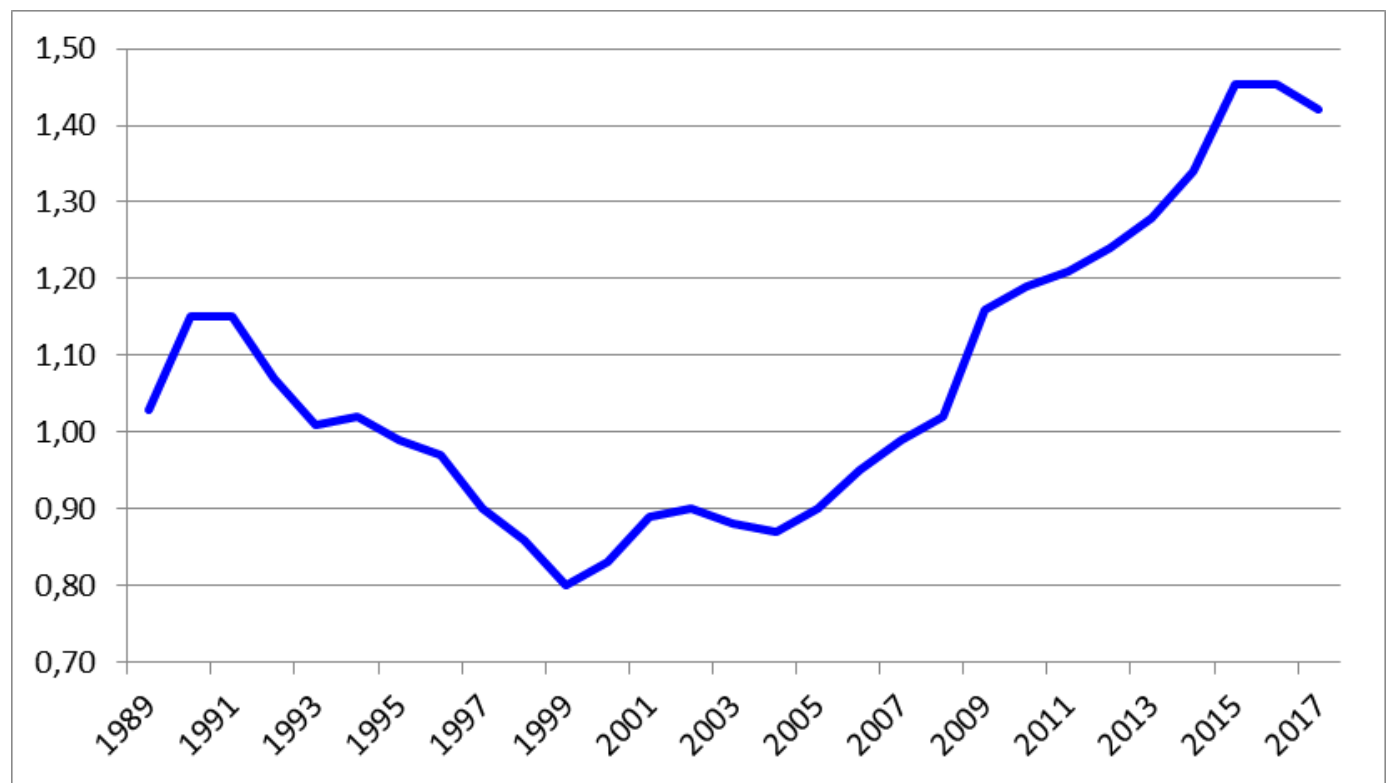

Fuente: Elaboración propia a partir de la información suministrada por la Jefa de la Oficina de Desarrollo Institucional del Conare, Mag. Xinia Morera González (comunicación personal, 21 de febrero, 2017).

Ahora bien, según lo señalado en el artículo 85 de la Constitución Política de la República, el cuerpo encargado de la coordinación de la educación superior universitaria estatal, es el ente responsable de la distribución de los recursos del FEES para cada universidad, este rol lo ha desarrollado el Conare. En ese contexto, el Conare ha acordado la distribución que se detalla en la Tabla 3. Como puede observarse, al monto total asignado al FEES se le deduce un 1,24\% destinado al financiamiento del Consejo Nacional de Rectores, de igual forma se deduce un 5,19\% para el llamado Fondo del Sistema, creado por acuerdo número 32-04 del Conare en el año 2004. El Fondo del Sistema procura fomentar la cultura de trabajo conjunto entre las instituciones universitarias estatales. Con estos recursos se brinda financiamiento a proyectos en los que participen académicos de al menos dos de las universidades miembros del Conare.

A partir de la creación de los Fondos del Sistema, los Rectores definieron áreas estratégicas para su desarrollo y, con base en ellas, han otorgado financiamiento a iniciativas como: la regionalización universitaria, la adquisición de equipo científico y tecnológico, la asignación de becas para funcionarios universitarios, la capacitación en inglés para estudiantes o el acceso a redes avanzadas de investigación. De igual forma, en razón de la 
importancia creciente de la educación a distancia para ampliar la cobertura y el impacto de la educación superior, su fortalecimiento se considera una inversión estratégica para la construcción del Sistema de la Educación Superior Universitaria Estatal, de ahí que parte de los Fondos del Sistema se dedicaran a este fin.

\section{Tabla 3}

Costa Rica: Distribución de recursos del FEES por Universidad. Vigente para el año 2017

\begin{tabular}{lc}
\hline Detalle & Porcentaje \\
\hline \multicolumn{2}{c}{ FEES Total } \\
Menos: Financiamiento del Conare & $1,24 \%$ \\
Fondos del Sistema & $5,19 \%$ \\
lgual: FEES Institucional & \\
Universidad de Costa Rica & $57,79 \%$ \\
Universidad Nacional de Costa Rica & $23,40 \%$ \\
Instituto Tecnológico de Costa Rica & $11,30 \%$ \\
Universidad Estatal a Distancia & $7,51 \%$ \\
\hline
\end{tabular}

Fuente: Elaboración propia a partir de la información suministrada por la Jefa de la Oficina de Desarrollo Institucional del Conare, Mag. Xinia Morera González (comunicación personal, 21 de febrero, 2017).

Según indica Cervantes, los acuerdos tomados por el Conare en el año 2004, una vez deducidos del monto total FEES lo correspondiente al financiamiento del Conare y los Fondos del Sistema, se obtiene el denominado FEES institucional. Ese es el monto que se distribuye a cada universidad con base en los porcentajes detallados en la Tabla 3 (Cervantes, 1999, p. 24).

Como puede apreciarse, en los datos analizados no aparece lo relativo a la Universidad Técnica Nacional (UTN). A pesar de que esta casa de enseñanza superior estatal fue creada en 2008, no es hasta 2015 que se toma el acuerdo de que forme parte del Conare. En materia de financiación, el acuerdo de adhesión de la UTN al Conare establece que:

[...] La incorporación de los recursos de la UTN no afectará el destino y pertenencia del FEES preexistente a este acuerdo de adhesión, el que se mantendrá contabilizado en forma separada para los efectos de su negociación, distribución, alcances y metas que sean acordadas en los convenios y acuerdos de financiamiento. Particularmente no afectará la meta establecida para dicho Fondo en el sentido de alcanzar el 1,5\% del PIB para el financiamiento de las cuatro universidades estatales a las que se destina constitucionalmente, según lo contemplado en el convenio de financiamiento suscrito para el quinquenio 2010-2015 [...]. (Conare, 2015, pp. 5-6) 
Es decir, la UTN se adhiere al Sistema, mas con sus propios recursos financieros, los cuales son brindados por el Estado, pero no forman parte del FEES. Para el año 2017 el presupuesto de la UTN representa 0,097\% del PIB.

\section{El caso de la Universidad de Costa Rica}

\subsection{Generalidades}

Mediante la aprobación de la Ley № 362 del 26 de agosto de 1940 se crea la Universidad de Costa Rica. Declarada en el 2001 institución Benemérita de la Patria, la Universidad de Costa Rica, según su Estatuto Orgánico:

Es una institución de educación superior y cultura, autónoma constitucionalmente y democrática, constituida por una comunidad de profesores y profesoras, estudiantes, funcionarias y funcionarios administrativos, dedicada a la enseñanza, la investigación, la acción social, el estudio, la meditación, la creación artística y la difusión del conocimiento. (Universidad de Costa Rica, 2005, p. 1)

La Universidad de Costa Rica es la institución de educación superior más antigua del país y la de mayor actividad académica en cuanto a cantidad de estudiantes y personal docente, número de opciones académicas en grado y posgrado, y cantidad de proyectos de investigación y vinculación con la sociedad. Diversos rankings ${ }^{3}$ publicados en los últimos años ubican a la Universidad de Costa Rica como la principal institución de educación superior de la Región Centroamericana y del Caribe y una de las más importantes de América Latina.

En la actualidad, la Universidad de Costa Rica cuenta con más de 40000 estudiantes, distribuidos en 12 sedes y recintos a lo largo del país. En la institución se imparten 240 opciones académicas a nivel de profesorado, diplomado, bachillerato y licenciatura. De igual forma, se ofertan 337 programas de posgrado. En esta misma línea, se desarrollan más de 711 proyectos de acción social en los 81 cantones del país y se realizan más de 1400 proyectos de investigación en las más diversas ramas del conocimiento.

${ }^{3}$ Ver, por ejemplo, https://www.topuniversities.com/university-rankings/world-university-rankings/2016 


\subsection{Financiamiento}

La Universidad de Costa Rica cuenta con dos fuentes básicas de financiamiento. De un lado, los denominados fondos corrientes, constituidos mayoritariamente por las transferencias del Estado vía FEES y el cobro de tasas por matrícula. De otro lado, los fondos provenientes del vínculo externo; de estos, una parte corresponde a transferencias de parte del Estado en cumplimiento de leyes específicas; otra parte, a los fondos del sistema y, finalmente, los provenientes de la vinculación remunerada con el sector externo. Datos extraídos de los estados financieros de la institución señalan que para el año 2016, el $86,46 \%$ de los ingresos corresponden a ingresos corrientes y un $13,54 \%$ a ingresos del vínculo externo. Del total de ingresos corrientes, un 1,33\% corresponde al cobro por tasas de matrícula, el cual, según la normativa vigente, debe invertirse de forma íntegra en el sistema de becas estudiantiles de la Institución.

Los recursos provenientes del vínculo externo comprenden todo tipo de vinculación de la Universidad de Costa Rica con el sector externo: programas o proyectos susceptibles de las dimensiones del quehacer académico (docencia, investigación y acción social), caracterizados por incluir el aporte económico del usuario en su financiamiento. Según la normativa institucional, que regula la vinculación con el sector externo, en esta relación debe prevalecer la solidaridad académica. Para ello, ha de contribuir con el desarrollo equilibrado de las áreas y dimensiones de la actividad sustantiva institucional, respetar los fines y propósitos de la Universidad y enriquecer las labores universitarias fundamentales mediante una relación estrecha con la sociedad.

Consecuentemente, se busca, en todo momento, el mejoramiento constante de la calidad de vida, el respeto integral de los derechos humanos, el desarrollo sostenible y el respeto a la autonomía universitaria en todos sus ámbitos. Desde el punto de vista de la administración financiera institucional, las actividades de vínculo externo se dividen en:

Empresas auxiliares: Están constituidas por todas aquellas acciones que generen recursos originados en la venta de bienes y servicios por parte de las unidades ejecutoras.

Fondos restringidos: Son todos aquellos aportes, donaciones o préstamos, así como los ingresos producto de lo estipulado en leyes específicas de la República y otros recursos provenientes del sector público, girados a favor de la Universidad de Costa Rica, para la realización de actividades o proyectos específicos cuyo uso está condicionado. Dentro de este grupo se contemplan los recursos que provienen del Fondo de Desarrollo Institucional, detallado adelante, así como los ingresos provenientes del Fondo del Sistema del Conare. 
Cursos especiales: Incluyen las actividades que forman parte del quehacer normal y sustantivo de la Institución, y que implican la venta de servicios por cursos de educación continua, actividades de carácter docente, de acción social e investigación. Surgen como resultado de necesidades concretas cuya cobertura es solicitada a la Universidad por parte de personas físicas o jurídicas que están dispuestas a financiar la totalidad de los gastos ocasionados por la Universidad como resultado de los servicios requeridos.

Programas de Posgrado con Financiamiento Complementario: Coexisten, en la institución, dos tipos de programas de posgrado. Por un lado, los que se consideran regulares y que son financiados con fondos corrientes como parte de las actividades docentes de las Unidades Académicas y, por otro lado, los programas de posgrado que son financiados fundamentalmente con los fondos que por concepto de matrícula cancelan sus estudiantes. Estos últimos son los denominados programas de posgrado con financiamiento complementario. Desde el punto de vista financiero, los ingresos generados por estos últimos se consideran como parte de la vinculación con el sector externo.

En relación con los fondos corrientes, tal y como se señaló en la sección anterior, las transferencias de Estado mediante el FEES se han incrementado considerablemente en los últimos años, especialmente a partir del año 2004 en que las tasas de crecimiento nominal anual, en promedio, han sido superiores al 15\%. Sin lugar a dudas, esa mejora en los ingresos ha permitido a las universidades estatales en general, y a la Universidad de Costa Rica en particular, contar con mejores condiciones para el desarrollo de su actividad sustantiva.

Esta coyuntura de incremento sostenido de ingresos por transferencias del Estado ha sido aprovechada por esta Casa de Enseñanza para implementar mejoras sustantivas al sistema de becas para estudiantes, ampliar de manera considerable la inversión en infraestructura y equipamiento y para atender las necesidades tecnológicas que permitan asegurar espacios idóneos para la producción académica.

A pesar de lo anterior, los recursos que aporta el Estado no son suficientes para el sostenimiento de las actividades sustantivas de la Universidad de Costa Rica. Es por ello que, en los últimos años, la institución ha fortalecido la vinculación remunerada con el sector externo mediante el establecimiento de políticas y lineamientos que permitan ordenar y estimular la generación de recursos y aprovechar el potencial universitario en la transferencia de conocimiento hacia los sectores productivos, económicos y sociales del país. 
Para tal fin, el Consejo Universitario emitió los "Lineamientos para la Vinculación Remunerada de la Universidad de Costa Rica con el Sector Externo". Estos señalan que la administración financiera de los recursos generados producto de la vinculación con sectores externos a la Institución se podrá desarrollar de acuerdo con los mecanismos institucionales, o bien, por medio de la Fundación Universidad de Costa Rica, un ente de carácter privado sin fines de lucro, creado como un mecanismo de promoción y gestión de los proyectos de investigación y acción social de la Universidad de Costa Rica.

En las condiciones actuales, según estos lineamientos, la vinculación remunerada con el sector externo, amplía la red de relaciones institucionales y demanda un reordenamiento interno, así como una definición de pautas organizativas que permitan asumir, coherente y responsablemente esta actividad, sin distorsionar los fines y la naturaleza misma de la institución. Bajo esa perspectiva, se establecieron políticas que permitan ordenar y estimular la vinculación remunerada con el sector externo, pero que, al mismo tiempo, garanticen que esta no entre en contradicción con las actividades sustantivas de la Universidad.

Según lo establecido por los Lineamientos para la Vinculación Remunerada de la Universidad de Costa Rica con el Sector Externo son las Facultades, Escuelas, Sedes regionales, Centros e Institutos de investigación, así como las Estaciones experimentales, las unidades operativas que ejecutan y generan las actividades de docencia, investigación y acción social de vinculación remunerada con el sector externo (Universidad de Costa Rica, 2017, p. 1).

Desde el punto de vista financiero, todo programa o proyecto de vinculación remunerada con el sector externo debe presentar a la vicerrectoría respectiva, para su aprobación definitiva, el presupuesto previamente aprobado por la dirección de la unidad operativa.

Del total de ingresos generados por el proyecto un 5\% será retenido con el propósito de que la institución cubra los costos administrativos generados por la gestión del proyecto. Adicionalmente, se crea un Fondo de Desarrollo Institucional, de manera que toda actividad de vinculación remunerada contribuirá con un $15 \%$ del total de los ingresos a este Fondo. El $80 \%$ restante será ejecutado directamente por las unidades operativas en el desarrollo del proyecto según el presupuesto aprobado. Una vez finalizado el proyecto, todos los excedentes serán administrados por las unidades operativas en las cuales se desarrolló la actividad del vínculo remunerado. 
Según la normativa, no estarán sujetas a la contribución al Fondo de Desarrollo Institucional las donaciones que reciban las unidades operativas, los recursos provenientes de aportes del Estado en cumplimiento de leyes específicas, así como aquellos proyectos declarados de interés institucional, según recomendación de la Vicerrectoría correspondiente, excepto en términos de reconocer hasta el $5 \%$ del costo por administración de los fondos.

\subsection{El Fondo de Desarrollo Institucional}

Como se señaló anteriormente, el Fondo de Desarrollo Institucional (FDI) es un fondo que se nutre de los recursos que, por concepto de recuperación de su inversión, asignan a la Universidad todos los proyectos de vinculación remunerada con el sector externo que realizan las unidades académicas. Según el Reglamento para la Administración del Fondo de Desarrollo Institucional, el propósito de este es contribuir con el desarrollo equilibrado de las áreas y dimensiones del quehacer universitario, dentro de principios de solidaridad y excelencia académica.

Es decir, este Fondo pretende potenciar el establecimiento de un sistema de solidaridad académica. Este sistema permite que parte de los recursos generados por áreas académicas, que por sus características tienen mayor facilidad para la vinculación externa remunerada, aporten a dicho Fondo y que con recursos de este se financien, entre otros, actividades de investigación y extensión en aquellas áreas en las que el financiamiento de la vinculación remunerada con el sector externo es poco probable.

Para la administración, uso y distribución del (FDI), el Reglamento para la Administración del Fondo de Desarrollo Institucional establece las disposiciones siguientes:

a. Un tercio (1/3) de los aportes al fondo por parte de cada unidad generadora se distribuirá de la siguiente forma: 60\% para estas y 40\% para la Decanatura o Dirección de Sede Regional. La Decanatura o Dirección de Sede, con el apoyo de su Consejo Asesor, decidirá sobre la utilización de ese 40\%, no obstante, los recursos deberán ser utilizados en la compra de materiales y suministros, maquinaria, equipo y construcciones, adiciones y mejoras de obras de infraestructura; y hasta un $20 \%$ de lo distribuido, para gastos que estimulen la consecución de fondos o que promuevan actividades de cooperación.

b. Los dos tercios (2/3) restantes se distribuirán de la siguiente manera: un $20 \%$ para crear un fondo permanente de capitalización, a cinco años plazo, que administrará la 
Oficina de Administración Financiera. Cumplido ese período, los intereses que, a partir de ese momento genere dicho fondo, se podrán utilizar en los proyectos de interés institucional, así definidos por la Rectoría. Un 30\% para programas de interés institucional, considerados así por la Rectoría, con base en las propuestas que sean presentadas por las Vicerrectorías y en el marco de las políticas y prioridades emanadas del Consejo Universitario. Un $50 \%$ se dividirá en partes iguales para el apoyo financiero de proyectos de investigación y para proyectos de acción social. Con estos recursos, se dará prioridad al financiamiento de los proyectos de investigación y acción social, en los que participen activamente estudiantes de grado y posgrado. (Universidad de Costa Rica, 2002, pp. 2-3)

La Figura 5 resume la forma en que se distribuye el denominado FDI.

\section{Figura 5}

Universidad de Costa Rica: Distribución del Fondo de

Desarrollo Institucional
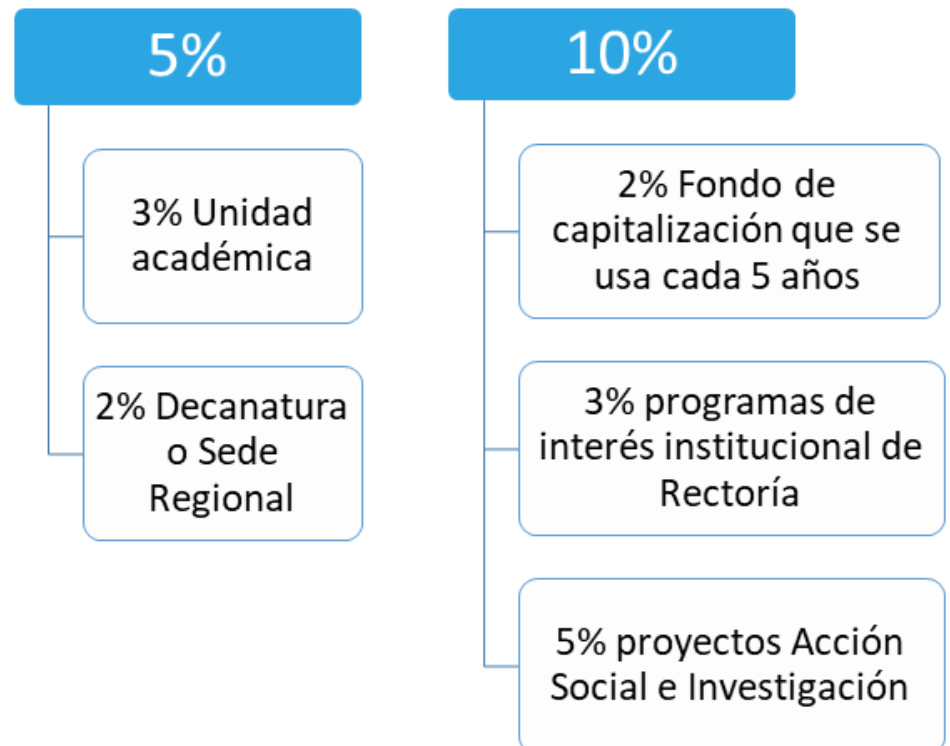

Fuente: Elaboración propia a partir del Reglamento para la Administración del Fondo de Desarrollo Institucional (Universidad de Costa Rica, 2002).

\section{Conclusiones}

En las políticas públicas del Estado costarricense, la educación ha jugado un papel primordial, al punto de que el financiamiento de todos sus niveles está consagrado en la Constitución Política de la República. En su artículo 78 se estableció, en primera instancia, 
que el Estado debía invertir el equivalente al 6\% del PIB en educación. Posteriormente, en el año 2011 este artículo fue reformado, de manera que esa cifra debe alcanzar el 8\% del PIB.

La situación fiscal del país ha impedido que el Estado cumpla con ese mandato constitucional; no obstante, para el año 2016 el porcentaje de inversión en educación alcanzó el 7,62\% del PIB, cifra que se encuentra muy por encima de los porcentajes de inversión mostrados por el resto de países de la región e, incluso, es mayor que el promedio de inversión en educación respecto al PIB que realizan los países miembros de la OCDE.

El alto nivel del déficit que muestran las finanzas públicas representa una seria amenaza a la posibilidad de que el Estado costarricense pueda mantener el nivel de inversión en educación y lograr el cumplimiento de lo establecido en el artículo 78 constitucional. De no lograrse, en el corto plazo, una reforma tributaria que permita al Estado recaudar más impuestos y una estrategia de contención del gasto público, día a día será mayor la cantidad de erogaciones por concepto de intereses y amortización de deuda que deberá atenderse, lo cual limitaría sensiblemente la posibilidad de mejorar las condiciones de financiamiento de la educación en general.

En materia de educación superior, el mecanismo de firma de convenios de financiamiento puesto en práctica desde el año 1989, generó, durante sus primeros años, una reducción considerable en el porcentaje de inversión respecto al PIB, posiblemente ligado a la difícil situación económica y a los programas de ajuste estructural a que fue sometido el Estado en esos años. No obstante, a partir de la firma del tercer y cuarto convenio de financiación, esta situación varía radicalmente, pues se pasa de un $0,8 \%$ en 1999 al 1,42\% del PIB en 2016. Según lo establecido en el quinto convenio de financiamiento, la inversión en educación superior alcanzará el 1,5\% del PIB en el momento en que se cumpla con el mandato constitucional de invertir del 8\% del PIB en educación. Esto colocaría a Costa Rica, al lado de los países del mundo que más invierten en educación superior pública estatal en relación con su producto interior bruto.

El Consejo Universitario de la Universidad de Costa Rica estableció lineamientos para regular y potenciar la generación de recursos por medio la vinculación remunerada con el sector externo. Como elemento innovador se estableció que todos los proyectos que generen recursos económicos deben contribuir con un $15 \%$ del total de sus ingresos a un Fondo de Desarrollo Institucional. Una parte de ese Fondo se utiliza para el desarrollo de proyectos institucionales estratégicos y otra parte se debe utilizar para el financiamiento de actividades de investigación y acción social. Esto, con la finalidad de brindar recursos a 
aquellas áreas académicas que, por su condición, se les hace difícil la vinculación remunerada con el sector externo.

En la Universidad de Costa Rica para el año 2016, el 86,5\% de su presupuesto proviene de transferencias del Estado y tasas por matrícula, mientras que un 13,5\% corresponde a la vinculación remunerada con el sector externo. Las tasas por matrícula representan el 1,33\% del total de los ingresos y, de acuerdo con la normativa institucional, la totalidad de estos recursos debe destinarse a la financiación parcial del sistema de becas estudiantiles.

Así, según la creciente necesidad de recursos para el desarrollo adecuado de su actividad sustantiva y la poca probabilidad de que el Estado pueda mantener constante las tasas de crecimiento de las transferencias hacia la institución, que se han dado en los últimos años, la Universidad de Costa Rica debe realizar esfuerzos por incrementar la vinculación remunerada con el sector externo como alternativa de financiamiento, sin que esto vaya en detrimento de las actividades docentes, de investigación y de acción social como ejes sustantivos de esta Casa de Estudios.

\section{Referencias}

Barquero, Jorge, Calvo, Elieth, Ortiz, Mariam, Quesada, Ileana, Valverde, Carla y Wachong, Verónica. (2006). Financiamiento de la Educación Superior: antecedentes y tendencias en el contexto nacional e internacional. San José, C.R.: Editorial Universidad de Costa Rica.

Cervantes, Flor. (1999). Propuesta de un mecanismo para establecer criterios de distribución del Fondo Especial de la Educación Superior Universitaria Estatal. Revista Educación, 23(1), 7-45.

Consejo Nacional de Rectores (Conare). (2013). Compendio de Leyes, Decretos y Convenios de la Educación Superior Universitaria Estatal en Costa Rica. [versión digital pdf]. Recuperado de https://www.conare.ac.cr/images/docs/leyes acuerdos/OPES 22013 Leyes convenios y decretos.pdf

Consejo Nacional de Rectores (Conare). (2015). Acta de la sesión 09-2015 [versión digital pdf]. Recuperado de http://servicios.conare.ac.cr/acuerdos/

Consejo Nacional de Rectores (Conare). (2015). Plan Nacional de la Educación Superior Universitaria Estatal 2016-2020. [versión digital pdf]. Recuperado de http://siesue.conare.ac.cr/images/documentos/planes 2016 2020.pdf

Costa Rica. (s.f.). Constitución Política de la República de Costa Rica [versión digital pdf]. Recuperado de http://pdba.georgetown.edu/Parties/CostaRica/Leyes/constitucion.pdf 
Estado de la Nación. (2016). Quinto informe del Estado de la educación. [versión digital pdf]. Recuperado de: http://www.estadonacion.or.cr/educacion2015/index.html

Fernández, Susana y Fernández, Sara. (2007). Evolución de la Educación Superior Costarricense: Un análisis de su modelo de financiación. Revista Actualidades Investigativas en Educación, 7 (2), 1-23. Doi: https://doi.org/10.15517/aie.v7i2.9267

Instituto Tecnológico de Costa Rica. (2016). Compendio Estadístico 2009-2015 [versión digital pdf]. Recuperado de https://www.tec.ac.cr/sites/default/files/media/doc/compendio-2009-2015-tec.pdf

Martínez, Bernal. (2016). Cronología de la Educación Costarricense. San José, C.R.: Imprenta Nacional.

Organización para la Cooperación y el Desarrollo Económico (OCDE). (s.f.). Estadísticas de países miembros. Recuperado de http://www.oecd.org/centrodemexico/estadisticas/

Sanyal, Bikas y Martín, Michaela. (2006). La financiación de la educación superior: perspectivas internacionales. En La Educación Superior en el mundo (pp.3-17). Barcelona. Mundiprensa.

Sauma, Pablo y Trejos, Juan Diego. (2014). Impacto de la política fiscal en la distribución del Ingreso y la pobreza en Costa Rica. Revista Ciencias Económicas, Universidad de Costa Rica, 32(2), 65-95. Doi: https://doi.org/10.15517/rce.v32i2.17259

Universidad de Costa Rica. (s.f.). Transparencia. UCR en cifras [versión digital pdf]. Recuperado de http://www.ucr.ac.cr/transparencia/

Universidad de Costa Rica. (2002). Reglamento para la Administración del Fondo de Desarrollo Institucional. Recuperado

en http://www.cu.ucr.ac.cr/normativ/fondo desarrollo.pdf

Universidad de Costa Rica. (2005). Estatuto Orgánico. Recuperado de http://www.cu.ucr.ac.cr/normativ/estatuto organico.pdf

Universidad de Costa Rica. (2017). Lineamientos para la vinculación remunerada de la Universidad de Costa Rica con el sector externo. Recuperado en http://www.cu.ucr.ac.cr/normativ/vinculacion sector externo.pdf

Universidad Estatal a Distancia (UNED). (2015). Informe de labores 2015 [versión digital pdf]. Recuperado de: http://www.uned.ac.cr/transparencia/images/documentos/informelabores/Informe Labor es 2015.pdf

Universidad Nacional de Costa Rica (UNA). (s.f.). Cifras UNA [versión digital pdf]. Recuperado de http://www.transparencia.una.ac.cr/index.php/categorias/una-en-cifras

Universidad Técnica Nacional (UTN). (2015). Universidad Técnica Nacional. De un Vistazo [versión digital pdf]. Recuperado de: http://www.utn.ac.cr/content/de-un-vistazo 